\title{
Unsupervised Window Extraction from Photogrammetric Point Clouds with Thermal Attributes
}

\author{
Dong Lin ${ }^{\text {a, } * \text {, Zhen Dong }}{ }^{\mathrm{b}}$, Xinlong Zhang ${ }^{\mathrm{a}}$, Hans-Gerd Maas ${ }^{\mathrm{a}}$ \\ a Technische Universität Dresden, Institute of Photogrammetry and Remote Sensing, Dresden, Germany - \\ (dong.lin, xinlong.zhang, hans-gerd.maas)@tu-dresden.de \\ ${ }^{\mathrm{b}}$ State Key Laboratory of Information Engineering in Surveying, Mapping and Remote Sensing, Wuhan University, China - \\ dongzhenwhu@whu.edu.cn
}

Commission II, WG II/4

KEY WORDS: Window extraction, point cloud, thermal attribute, segmentation, energy optimization, feature extraction

\begin{abstract}
:
The automatic extraction of windows from photogrammetric data has achieved increasing attention in recent times. An unsupervised windows extraction approach from photogrammetric point clouds with thermal attributes is proposed in this study. First, point cloud segmentation is conducted by a popular workflow: Multiscale supervoxel generation is applied to the image-based 3D point cloud, followed by region growing and energy optimization using spatial positions and thermal attributes of the raw points. Afterwards, an object-based feature (window index) is extracted using the average thermal attribute and the size of the object. Next, thresholding is applied to extract initial window regions. Finally, several criterions are applied to further refine the extraction results. For practical validation, the approach is evaluated on an art nouveau building row façade located at Dresden, Germany.
\end{abstract}

\section{INTRODUCTION}

An increased interest in point cloud segmentation and window extraction from point cloud data has emerged in recent years. In order to extract structures (e.g. windows) from a raw unstructured point cloud, one popular approach is point cloud segmentation followed by feature extraction. Point cloud segmentation, which merges adjacent points or voxels into subsets using one or more criterions (e.g. similar normal vectors, similar attributes), provides basic meaningful regions (e.g. planar) for further detection and recognition. After segmenting the whole façade point cloud into dozens of regions, feature extraction is needed to describe the characteristics of different objects (e.g. windows, doors) and thus is helpful for further target detection and recognition.

Point Cloud Segmentation: The extensive work targeted on point cloud segmentation in the last years can roughly be classified to four categories: model fitting, region growing, feature clustering and energy optimization.

Model fitting extracts connected points by fitting their geometric features (e.g. positions and normal vectors) into a local or global geometric model. Specifically, points satisfying the same geometric model fitting criteria are extracted from the whole point cloud as one segment. The most commonly used approaches are random sample consensus (RANSAC) and Hough transform (HT). RANSAC first generates several models by randomly sampling points, then the model with the largest set of inliers is chosen (Fischler and Bolles, 1981; Schnabel, 2007). HT first transforms each point into a parameter space according to the geometric expression, then the segments are extracted by selecting the local maxima in the accumulator space using a voting-strategy (Ballard, 1981; Vosselman et al.,
2004). Although RANSAC and HT are able to extract meaningful geometric primitives (i.e. planes, spheres, cylinders, cones) from point cloud with noises, they are sensitive to parameter selection (e.g. threshold for inliers). In addition, applying RANSAC for multiple plane detection is not only computationally expensive, but will also often deliver suboptimal results (e.g. inaccurate first plane detection would negatively affect the accuracy of the following planes) (Pham et al., 2016).

Region growing (RG) extract segments by iteratively merging adjacent points or voxels with similar features (e.g. normal vector, curvature). Point-based RG (Tóvári and Pfeifer, 2005), voxel-based RG (Deschaud and Goulette, 2010), octree-based RG (Vo et al., 2015) and hybrid RG (Xiao et al., 2013) have been extensively investigated and discussed for point cloud segmentation. Most of these methods start picking up some points or voxels as seeds, then the neighbours of the seeds are merged when these neighbours satisfy the predefined criterion (e.g. similar normal vector). A main benefit of these approaches is the high computational efficiency, while a major drawback is that they are sensitive to noise and varying point density. Furthermore, segmentation results largely depend on the growing criteria and the selection of the seeds.

Similar to RG, feature clustering is also a process of grouping points with similar features into a cluster under common constraints. K-means (Shi et al., 2011), fuzzy C-means (Biosca and Lerma, 2008), hierarchical clustering (Xu et al., 2018) are frequently adopted for segmentation task of the point clouds. Compared with RG, one advantage of such approaches is that no seeds are needed for initialization. However, such approaches are also not robust to noise and outliers due to the

\footnotetext{
* Corresponding author
} 
difficulty in definition of the neighbourhood and the chosen clustering criterion.

Energy optimization formulates the segmentation issue as an energy function minimization problem and is widely used with both 2D image and 3D point cloud data (Isack and Boykov, 2012; Pham et al., 2016; Pham et al., 2018; Dong et al., 2018). Yan et al. (2014) formulated the roof segmentation task from airborne laser scanner point clouds as an energy minimization problem and adopted $\alpha$-expansion approach (Delong et al., 2012) to minimize the function. Dong et al. (2018) implemented a guided sampling algorithm to optimize the global energy function in a mutually reinforcing manner and mainly targeted on façade point clouds. Wang et al. (2016) utilized a similar energy optimization approach for multiple plane detection in indoor point clouds. When compared to other methods, the main benefit of energy optimization approaches is they are rather robust to noise. However, the main drawback is they are computationally expensive and dependent on initial planes.

Window Extraction: Windows, as basic elements of facades, play an important role in various applications. Windows belong to openings in CityGML standard with five levels of details (LoD). Specifically, LoD3 requires detailed modelling of windows. And rows of windows can be used to determine the number of floors. In energy inspection of buildings, the ratio of windows to the façade area is important to estimate the energy consumption of buildings. Windows are often regarded as the main possibility as thermal leakage potentials. Therefore, automatic window detection is of vital importance for thermal inspection of buildings. In general, there are two main strategies available in existing publications: pattern recognition and holebased methods.

Pattern recognition takes advantage of shape grammar to detect symmetries and repetitive structures for window or door extraction. Pauly et al. (2008) detected repetitive patterns based on accumulating local symmetry votes in the transformation space. Pairwise similarity transformation is conducted in a regular grid. Then an aggregation procedure is conducted to detect rotational and cylindrical grids, helices, and spirals. Although this approach is able to estimate missing data, it is computationally expensive and sensitive to the calculation of curvatures. Friedman and Stamos (2013) proposed a real-time $3 \mathrm{D}$ registration procedure during data acquisition. Utilizing this approach, they employed vertical periodicity to detect window groups with similar widths for high-rise buildings. However, this approach might fail in low-rise residential structures where vertically repetitive features are unlikely to contain. Becker (2009) combined data-driven and model-driven strategies to reconstruct façade elements using grammar rules, which is robust to incomplete data and erroneous data.

Windows, which have texture-less, transparent and highlyreflective surfaces, could introduce large artifacts and cause data gaps in point clouds acquired by terrestrial laser scanning (TLS). Therefore, another popular approach to extract windows is hole-based method. Pu and Vosselman (2009), Truong-Hong et al. (2011) implemented Triangular Irregular Network (TIN) based approaches for window extraction. The basic principle of these approaches is the triangles with long sides correspond to boundary points where holes exist. More recently, Zolanvari and Laefer (2016) presented a slicing method (SM) to detect openings and boundary points of facades. Given the presegmented facades, openings could be obtained by slicing the façade into strips horizontally and vertically, projecting the boundary points into one dimension, and searching for gaps. Later, Zolanvari et al. (2018) extended the SM approach for full $3 \mathrm{D}$ structures and sloped roofs.

As shown above, most of the existing approaches focus on only utilizing geometric features from given point clouds and ignore other attributes. Although $\mathrm{Pu}$ and Vosselman (2009) reported that integrating RGB information cannot guarantee to achieve better segmentation results than solely using spatial position information, in this study, we try to combine attributes obtained from a thermal camera with geometric features for point cloud segmentation and window extraction.

\section{DATA DESCRIPTION}

The 3D point cloud used in this paper is generated using the approach described by (Lin et al., 2019). The principle is as follows: Firstly, a RGB image sequence is used for reference 3D point cloud generation using structure from motion (SfM) tools (e.g. Agisoft PhotoScan $\left.{ }^{\circledR}\right)$. Next, a thermal point cloud, which is generated by a thermal image sequence, is registered to the RGB point cloud using a coarse point cloud registration followed by a fine image-based registration. Afterwards, a global image pose refinement is applied to remove blur effects and to increase consistency in texture mapping results. An example of a generated photogrammetric point cloud with thermal attributes is shown in Figure 1.

Although the data are equipped with geometric features (e.g. XYZ coordinates), RGB attributes and thermal attributes, this paper focuses on segmenting the point cloud and extracting windows using only geometric features and thermal attributes. Note that achieving accurate thermal attributes also requires a radiometric calibration approach (Lin et al., 2018). Figure 1 shows the colour-coded temperature distribution of the entire building facade. In this paper, these thermal attributes, linearly scaled to 8 bit as temperature derived RGB values, are used for later segmentation and extraction works. The reason why we use temperature derived RGB values rather than real temperature values $\left({ }^{\circ} \mathrm{C}\right)$ for segmentation procedure, is the input requirements of the used supervoxel generation algorithm, which asks for three 8 bit scalars as inputs. An energy optimization based segmentation followed by an object-based extraction approach is proposed in this study.

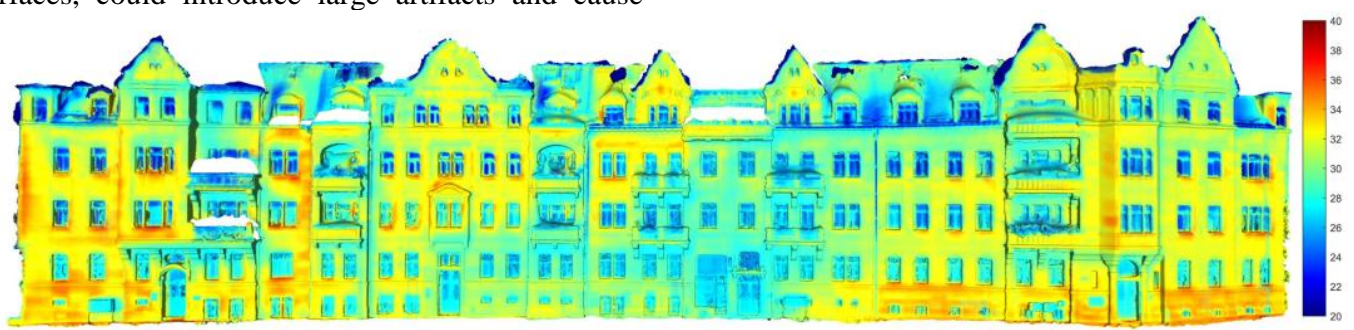

Figure 1. Projection of a 3D point cloud of a façade with thermal attributes $\left({ }^{\circ} \mathrm{C}\right)(\mathrm{Lin}$ et al., 2019) 


\section{METHODOLOGY}

Similar to the workflow implemented in Dong et al. (2018), the segmentation approach implemented in this study includes three main steps: multiscale supervoxel generation, region growing and energy optimization.

Multiscale Supervoxel Generation: Multiscale supervoxel generation starts to oversegment the point cloud into supervoxels at the largest scale $\left(r_{\max }\right)$, then the supervoxels are classified as planar or nonplanar according to saliency features. Afterwards, the nonplanar supervoxels are further segmented into supervoxels at a smaller scale $(r=r * \Delta r)$. The algorithm repeats such steps until the scale parameter $r$ is less than the preset minimum scale parameter $\left(r_{\text {min }}\right)$.

The voxel cloud connectivity segmentation (VCCS) method (Papon et al., 2013) is applied here to over-segment the point clouds into supervoxels at different scales. Specifically, spatial distance, normal vector deviation and colour distance (here applied to the thermal attributes) are considered during supervoxel generation. And the weights of spatial distance, normal vector deviation and colour distance are set equal. Furthermore, the planar determination criterion for each supervoxel is as follows:

First, a covariance matrix is calculated using all points of the supervoxel. Then eigenvalues $\left(\lambda_{1}>\lambda_{2}>\lambda_{3}\right)$ and the corresponding eigenvectors $\left(e_{1}, e_{2}, e_{3}\right)$ are computed using principle component analysis. Afterwards, the saliency features are calculated using equation (1). Finally, the supervoxel is classified as planar or nonplanar according to equation (2). Note that $n_{m}, n_{s}$ represent normal vector of point $m$ and normal vector of supervoxel $s$, respectively. $N$ denotes the number of the points in supervoxel $s . \overline{\mathrm{n}_{s}}$ represents the average angle between the normal vectors of all the inlier points and the normal vector of their supervoxel. As photogrammetric point clouds are usually more noisy than TLS point clouds, $\overline{\mathrm{n}_{s}}$ should be taken into consideration during planar determination. Multiscale supervoxel generation results are shown in Figure 2.

$$
\left\{\begin{array}{c}
m_{1}=\frac{\sqrt{\lambda_{1}}-\sqrt{\lambda_{2}}}{\sqrt{\lambda_{1}}} \\
m_{2}=\frac{\sqrt{\lambda_{2}}-\sqrt{\lambda_{3}}}{\sqrt{\lambda_{1}}} \\
m_{3}=\frac{\sqrt{\lambda_{3}}}{\sqrt{\lambda_{1}}} \\
\overline{\mathrm{n}_{s}}=\frac{1}{N} \sum_{m=1}^{N}\left|\operatorname{acos}\left(n_{m} \cdot n_{s}\right)\right| \\
\quad \text { if } m_{2}>m_{1} \cap m_{2}>m_{3} \cap \overline{\mathrm{n}_{s}}<5^{\circ}
\end{array}\right.
$$

After multiscale supervoxel generation, planar regions and individual points serve as basic units for further region growing and energy optimization. For each basic unit, statistical features, which are used in later segmentation steps, are calculated by equation (3).

$$
\left\{\begin{array}{c}
n_{s}\left(a_{s}, b_{s}, c_{s}\right)=e_{3} \\
d_{s}=-\left(a_{s} x_{s}+b_{s} y_{s}+c_{s} z_{s}\right) \\
\operatorname{thermal}\left(R_{s}, G_{s}, B_{s}\right) \\
f_{s}=\frac{\lambda_{3}}{\lambda_{1}+\lambda_{2}+\lambda_{3}}
\end{array}\right.
$$

where $n_{s}\left(a_{s}, b_{s}, c_{s}\right)$ represents the normal vector of basic unit $s$, $\left(x_{s}, y_{s}, z_{s}\right)$ is the centre position of basic unit $s, d_{s}$ is the distance from origin to the implicit plane, thermal $\left(R_{S}, G_{S}, B_{S}\right)$ represent the average thermal attribute of all the inlier points in basic unit $s$. Such temperature derived RGB colours are utilized for further segmentation. $f_{s}$ is the curvature of the basic unit $s$. Note that supervoxels take advantage of all their inlier points to calculate the covariance matrix, while individual points consider their $k$ adjacent points to compute the covariance matrix.

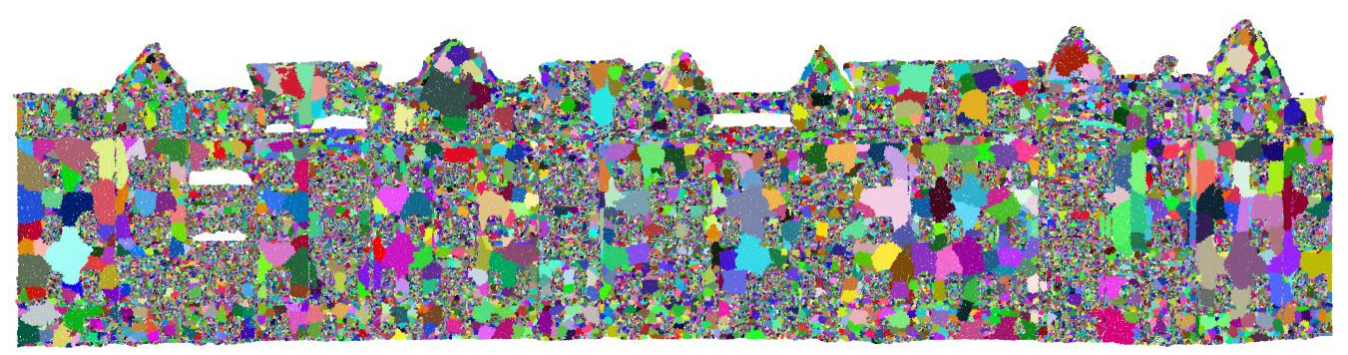

(a)

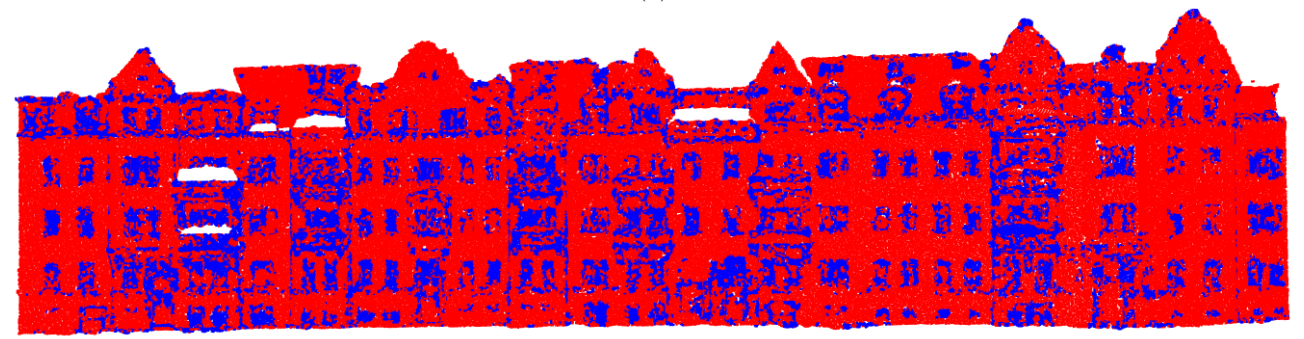

(b)

Figure 2. Multiscale supervoxel generation. (a) Multiscale supervoxels (b) Multiscale planar classification results. Red points belong to planar regions while blue points refer to non-planar points. 
Region Growing: A hybrid region growing is implemented here to generate initial planes for the later energy optimization procedure. The algorithm gradually merges adjacent basic units with similar statistical features to generate a number of planes. The basic workflow is as follows:

Region growing starts at the basic unit with the minimum curvature. Then the neighbouring basic units, which have similar normal vector $n(a, b, c)$, similar thermal attributes $(R, G, B)$ and a similar implicit plane distance $d$, is merged into current plane. In this case, angle criterion for normal vector deviation is $10^{\circ}$, implicit plane distance deviation criterion is $0.2 \mathrm{~m}$ and thermal attributes deviation threshold is 60 (8 bit). The repeated merging process is performed with the depth-first search (DFS). DFS explores the potential unmerged basic units as far as possible to generate one plane. If no more satisfying basic units are found, the DFS will start from another unsegmented basic unit with the lowest curvature and repeat the searching process. Note that the neighbouring basic units are defined using radius consideration rather than $k$ adjacent. In addition, the minimum number of inlier points for a valid plane is set to $\kappa$ (e.g. 20); thus, those planes, which have inliers lower than $\kappa$, are removed during region growing. The hybrid region growing result is shown in Figure 3. All the segments generated by hybrid region growing are taken as initial inputs for energy optimization process.

Energy Optimization: In this part, point cloud segmentation is formulated as an energy function minimization problem shown in equation (4).

$\mathrm{E}(S, P)=\overbrace{\sum_{s \in S, p \in P} \operatorname{dis}(s, p)}^{\text {Data Cost }}+\overbrace{\sum_{\left(s_{1}, s_{2}\right) \in \mathrm{edge}} \delta\left(s_{1}, s_{2}\right)}^{\text {Smooth Cost }}+\overbrace{\kappa \cdot|P|}^{\text {Label Cost }}$

The energy function is formed by data cost, smoothness cost and label cost. In this study, the data cost term measures spatial distance and thermal attribute distance between the basic units and the planes, which is shown in equation (5).

$$
\left\{\begin{array}{c}
\operatorname{dis}(s, p)=\left(\frac{s_{-} d i s}{\sigma_{s}}\right)^{2}+\left(\frac{t_{-} d i s}{\sigma_{t}}\right)^{2} \\
s_{-} d i s=\sum_{m \in S} \frac{\left|a_{p} \cdot x_{m}+b_{p} \cdot y_{m}+c_{p} \cdot z_{m}+d_{p}\right|}{\sqrt{a_{p}^{2}+b_{p}{ }^{2}+c_{p}{ }^{2}}} \\
t_{-} \text {dis }=\sum_{m \in S} \sqrt{\left(R_{m}-R_{p}\right)^{2}+\left(G_{m}-G_{p}\right)^{2}+\left(B_{m}-B_{p}\right)^{2}}
\end{array}\right.
$$

where $m$ represents the inlier point of the basic unit $s$. Spatial distance between basic unit $s$ and plane $p$ sums up all the spatial distances between the inlier points $m$ and the plane $p$. Similarly, thermal attribute distance sums up all the colour distances between the inlier points $m$ and the plane $p . \sigma_{s}$ is the weight parameter for spatial distance, while $\sigma_{t}$ is the weight parameter of colour distance. As shown in equation (5), the lower the spatial and colour distance between the basic unit $s$ and the plane $p$, the higher the possibility of merging $s$ into $p$ is

The smoothness cost, shown in equation (4), encourages the adjacent basic units to have the same labels, i.e. to belong to the same plane. A TIN is constructed to define the neighbourhood system for all of the basic units. Note that neighbouring basic units means they are connected by an edge line in the TIN. Potts model (Boykov et al., 2001) is selected to describe the smoothness cost for each pair of the neighbouring basic units, which is shown in equation (6). Thus, if the neighbouring basic units $s_{1}$ and $s_{2}$ belong to the same plane, the smoothness cost $\delta\left(s_{1}, s_{2}\right)$ is 0 . Otherwise, $\delta\left(s_{1}, s_{2}\right)$ is set to 1 .

$$
\delta\left(s_{1}, s_{2}\right)= \begin{cases}0 & \text { if } L_{s_{1}}=L_{s_{2}} \\ 1 & \text { if } L_{s_{1}} \neq L_{S_{2}}\end{cases}
$$

The label cost, shown in equation (4), penalizes the number of planes. $\kappa$ is the minimum point number for a plane, $|P|$ represents the number of the generated planes after segmentation. This term aims to reduce the number of oversegmented planes.

The energy function minimization process is implemented in an iterative manner and terminates if the energy function is not decreased anymore. During each iteration, statistical features of all the planes are re-computed using their newly derived inlier points. The energy optimization result is shown in Figure 4.

Window extraction: After segmentation, objects are obtained. Thus, object-based features could be calculated (e.g. objectbased thermal features could be computed by averaging all the thermal attributes of points in the objects). The size feature of an object is calculated according to equation (7).

$$
\text { Size }=\Delta \mathrm{X}+\Delta \mathrm{Y}+\Delta \mathrm{Z}
$$

where $\Delta \mathrm{X}, \Delta \mathrm{Y}$ and $\Delta \mathrm{Z}$ correspond to the bounding box of an object in $\mathrm{X}, \mathrm{Y}, \mathrm{Z}$ axis respectively. As shown in the segmentation result (Figure 4), window objects usually have smaller scales than other objects (e.g. wall objects).

Windows in thermal images will usually reflect objects nearby due to mirror-reflection effects. Specifically, from a terrestrial point of view, most of the windows, especially those located at relatively higher levels of the building, reflect the cold sky, thus windows will usually have lower temperatures compared to walls. Furthermore, the higher temperature derived B (blue) value and smaller Size value an object has, the more likely the object will be a window object. Thus, a window index (WI) is defined as equation (8).

$$
\mathrm{WI}=\frac{\mathrm{B}}{\text { Size }}
$$

For the whole point cloud, object-based WI feature is illustrated in Figure 5.

Thus, several criterions are designed and implemented to generate windows extraction results. First, a simple threshold of window index (e.g. more than 15) is applied to extract windows from the point clouds. Afterwards, as the normal vectors of the windows are considered to be almost parallel to the $\mathrm{Z}$ axis, a constraint is to set a threshold for the intersection angle between the normal vector of initial windows objects and axis $\mathrm{Z}$ (e.g. lower than $20^{\circ}$ ). Then, objects with relatively large area (e.g. more than $3 \mathrm{~m}^{2}$ ), which almost stand on the ground (e.g. distance to the lowest part of the building model lower than $0.3 \mathrm{~m})$, are considered to be doors rather than windows. Thus, the extraction result is shown in Figure 6. 


\section{DISCUSSIONS AND CONCLUSION}

To evaluate the window extraction result, correctness and completeness are used, which are shown in equation (9) and (10).

$$
\begin{gathered}
\text { correctness }=\frac{\|T P\|}{\|T P\|+\|F P\|} \\
\text { completeness }=\frac{\|T P\|}{\|T P\|+\|F N\|}
\end{gathered}
$$

where true positive (TP) is a window region and is detected as a window, false positive (FP) is a non-window region however is detected as a window, and false negative (FN) is a window region but is not detected as a window. The ground truth (bounding boxes of the windows) is measured manually. Similar to the work (Malihi et al., 2018), if at least $70 \%$ of points in a segment are located inside a reference bounding box, this segment is considered to be a TP. While if more than $50 \%$ of points in a segment are not located inside a reference bounding box, this segment is counted as a FP. If a reference bounding box has no corresponding segment or at least $50 \%$ of points in a segment, this is considered to be a FN. The evaluation is shown in Table 1.

\begin{tabular}{|c|c|c|c|c|}
\hline TP & FP & FN & Correctness & Completeness \\
\hline 98 & 22 & 17 & $82 \%$ & $85 \%$ \\
\hline
\end{tabular}

Table 1. Correctness and Completeness of window extraction result

As shown in Table 1, the window extraction result shows a Correctness rate of $82 \%$ and a Completeness percentage of $85 \%$. One assumption this method relies on is significant temperature differences between windows and their neighbouring objects (e.g. wall). This is the reason why some windows were not recognized, for instance due to the reflections of the nearby warm objects, such differences are largely moderated, which increases the difficulty in extracting windows using our approach. In future work, the wrongly extracted segments, which do not belong to windows, should be removed by utilizing shape-based rules and topology analysis.

\section{ACKNOWLEDGEMENTS}

The research work in this paper has been funded by China Scholarship Council (No. 201603170234).

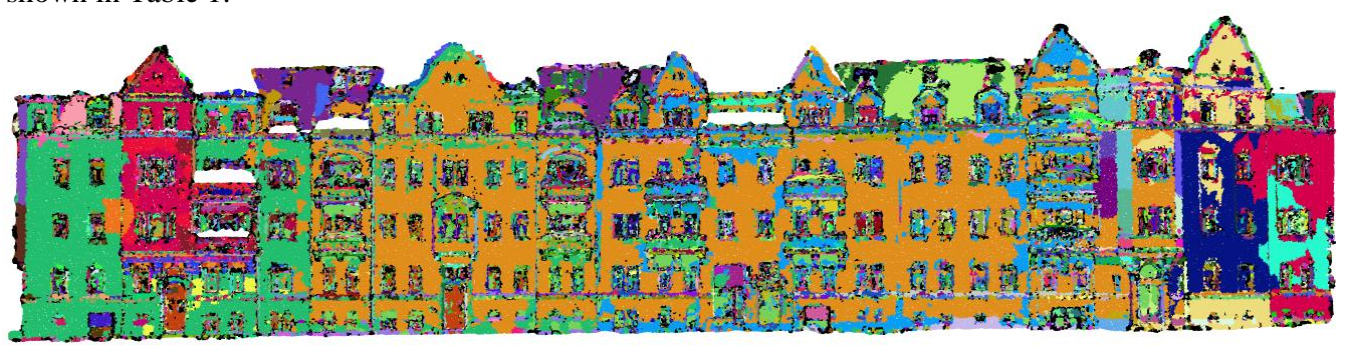

Figure 3. Hybrid region growing result

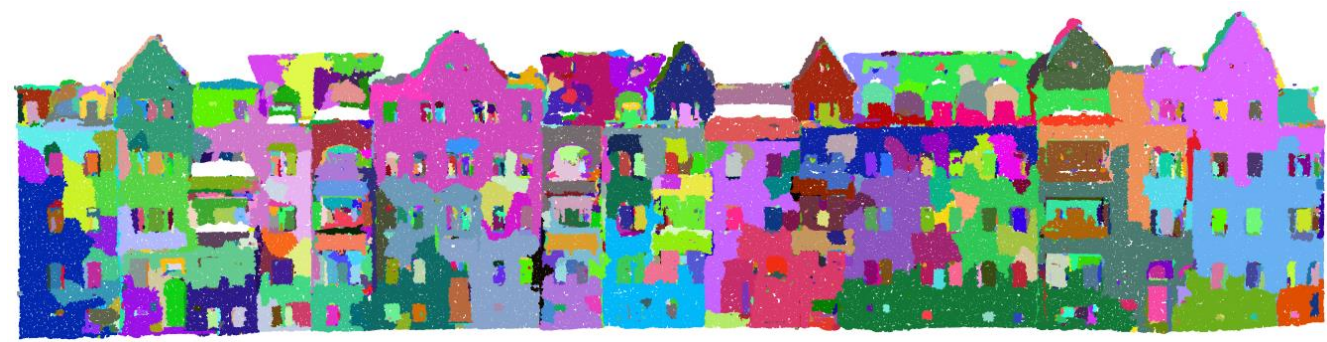

Figure 4. Energy optimization result

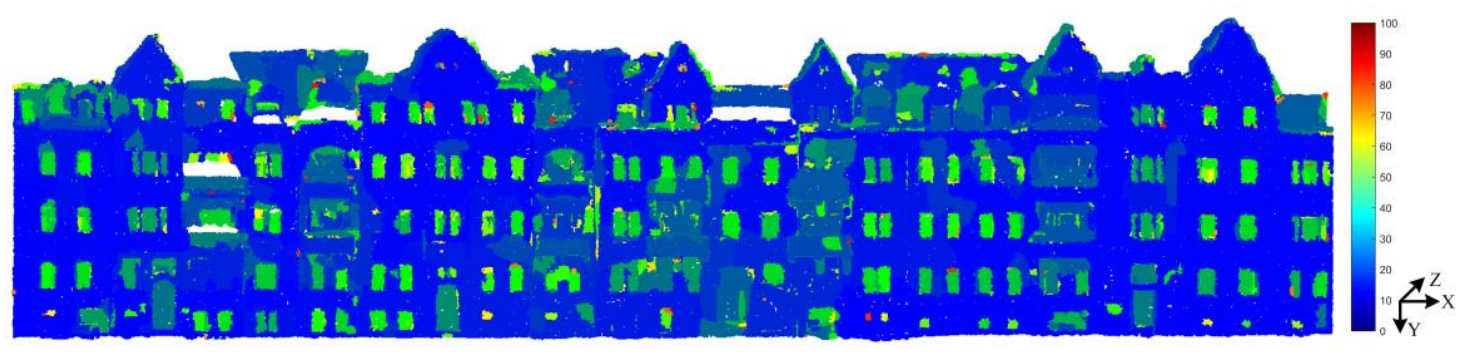

Figure 5. Objected-based window index feature. 


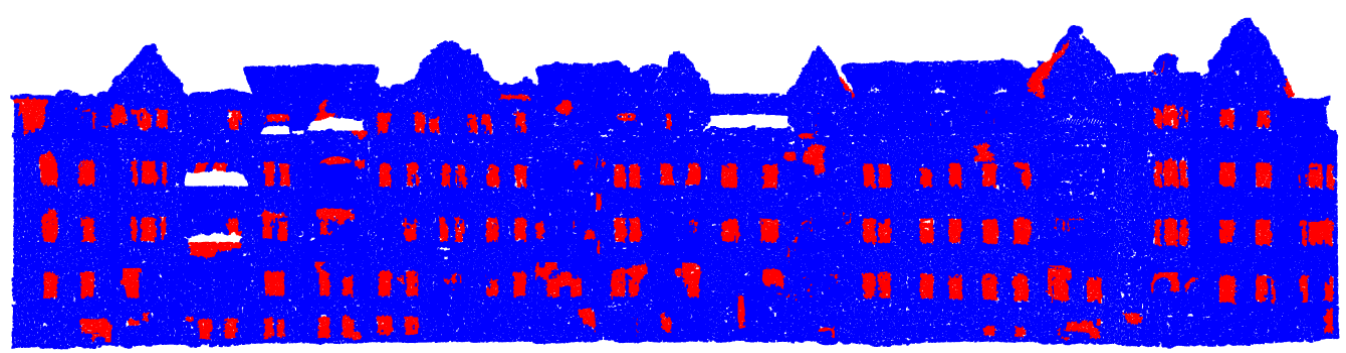

Figure 6. Window extraction result. Red segments refer to windows regions.

\section{REFERENCES}

Ballard, D.H., 1981. Generalizing the Hough transform to detect arbitrary shapes. Pattern Recognition, 13(2): 111-122.

Becker, S., 2009. Generation and application of rules for quality dependent façade reconstruction. ISPRS Journal of Photogrammetry and Remote Sensing, 64(6), pp.640-653.

Boykov, Y., Veksler, O. and Zabih, R., 2001. Fast approximate energy minimization via graph cuts. IEEE Transactions on pattern analysis and machine intelligence, 23(11), pp.12221239.

Biosca, J.M. and Lerma, J.L., 2008. Unsupervised robust planar segmentation of terrestrial laser scanner point clouds based on fuzzy clustering methods. ISPRS Journal of Photogrammetry and Remote Sensing, 63(1), pp.84-98.

Delong, A., Osokin, A., Isack, H.N. and Boykov, Y., 2012. Fast approximate energy minimization with label costs. International journal of computer vision, 96(1), pp.1-27.

Deschaud, J.E. and Goulette, F., 2010, May. A fast and accurate plane detection algorithm for large noisy point clouds using filtered normals and voxel growing. In 3DPVT.

Dong, Z., Yang, B., Hu, P. and Scherer, S., 2018. An efficient global energy optimization approach for robust 3D plane segmentation of point clouds. ISPRS Journal of Photogrammetry and Remote Sensing, 137, pp.112-133.

Fischler, M.A., Bolles, R.C., 1981. Random sample consensus: a paradigm for model fitting with applications to image analysis and automated cartography. Communications of the ACM, 24 (6), 381-395.

Friedman, S. and Stamos, I., 2013. Online detection of repeated structures in point clouds of urban scenes for compression and registration. International journal of computer vision, 102(1-3), pp.112-128.

Kazhdan, M. and Hoppe, H., 2013. Screened poisson surface reconstruction. ACM Transactions on Graphics (ToG), 32(3), p.29.

Lin, D., Maas, H.G., Westfeld, P., Budzier, H. and Gerlach, G., 2018. An advanced radiometric calibration approach for uncooled thermal cameras. The Photogrammetric Record, 33(161), pp.30-48.

Lin, D., Jarzabek-Rychard, M., Tong, X.C. and Maas, H.-G. 2019. Fusion of Thermal Imagery with Point Clouds for
Building Façade Thermal Attribute Mapping. ISPRS Journal of Photogrammetry and Remote Sensing, 151, pp.162-175.

Malihi, S., Valadan Zoej, M., Hahn, M. and Mokhtarzade, M., 2018. Window Detection from UAS-Derived Photogrammetric Point Cloud Employing Density-Based Filtering and Perceptual Organization. Remote Sensing, 10(8), p.1320.

Papon, J., Abramov, A., Schoeler, M. and Worgotter, F., 2013. Voxel cloud connectivity segmentation-supervoxels for point clouds. In Proceedings of the IEEE conference on computer vision and pattern recognition (pp. 2027-2034).

Pauly, M., Mitra, N.J., Wallner, J., Pottmann, H. and Guibas, L.J., 2008. Discovering structural regularity in 3D geometry. ACM transactions on graphics (TOG), 27(3), p.43.

Pham, T.T., Do, T.T., Sünderhauf, N. and Reid, I., 2018, May. Scenecut: Joint geometric and object segmentation for indoor scenes. In 2018 IEEE International Conference on Robotics and Automation (ICRA) (pp. 1-9). IEEE.

Pham, T.T., Eich, M., Reid, I. and Wyeth, G., 2016, October. Geometrically consistent plane extraction for dense indoor 3D maps segmentation. In Intelligent Robots and Systems (IROS), 2016 IEEE/RSJ International Conference on (pp. 4199-4204). IEEE.

$\mathrm{Pu}, \quad$ S. and Vosselman, G., 2009. Knowledge based reconstruction of building models from terrestrial laser scanning data. ISPRS Journal of Photogrammetry and Remote Sensing, 64(6), pp.575-584.

Isack, H. and Boykov, Y., 2012. Energy-based geometric multimodel fitting. International journal of computer vision, 97(2), pp.123-147.

Schnabel, R., Wahl, R. and Klein, R., 2007, June. Efficient RANSAC for point cloud shape detection. In Computer graphics forum (Vol. 26, No. 2, pp. 214-226). Oxford, UK: Blackwell Publishing Ltd.

Shi, B.Q., Liang, J. and Liu, Q., 2011. Adaptive simplification of point cloud using k-means clustering. Computer-Aided Design, 43(8), pp.910-922.

Truong-Hong, L., Laefer, D.F., Hinks, T. and Carr, H., 2011. Flying voxel method with Delaunay triangulation criterion for façade/feature detection for computation. Journal of Computing in Civil Engineering, 26(6), pp.691-707.

Tóvári, D. and Pfeifer, N., 2005. Segmentation based robust interpolation-a new approach to laser data filtering. 
International Archives of Photogrammetry, Remote Sensing and Spatial Information Sciences, 36(3/19), pp.79-84.

Vo, A.V., Truong-Hong, L., Laefer, D.F. and Bertolotto, M., 2015. Octree-based region growing for point cloud segmentation. ISPRS Journal of Photogrammetry and Remote Sensing, 104, pp.88-100.

Vosselman, G., Gorte, B.G., Sithole, G. and Rabbani, T., 2004. Recognising structure in laser scanner point clouds. International archives of photogrammetry, remote sensing and spatial information sciences, 46(8), pp.33-38.

Wang, L., Shen, C., Duan, F. and Guo, P., 2016, October. Energy-Based Multi-plane Detection from 3D Point Clouds. In International Conference on Neural Information Processing (pp. 715-722). Springer, Cham.

Xiao, J., Zhang, J., Adler, B., Zhang, H. and Zhang, J., 2013. Three-dimensional point cloud plane segmentation in both structured and unstructured environments. Robotics and Autonomous Systems, 61(12), pp.1641-1652.

Xu, Y., Yao, W., Tuttas, S., Hoegner, L. and Stilla, U., 2018. Unsupervised Segmentation of Point Clouds From Buildings Using Hierarchical Clustering Based on Gestalt Principles. IEEE Journal of Selected Topics in Applied Earth Observations and Remote Sensing.

Yan, J., Shan, J. and Jiang, W., 2014. A global optimization approach to roof segmentation from airborne lidar point clouds. ISPRS Journal of Photogrammetry and Remote Sensing, 94, pp.183-193.

Zolanvari, S.I. and Laefer, D.F., 2016. Slicing Method for curved façade and window extraction from point clouds. ISPRS Journal of Photogrammetry and Remote Sensing, 119, pp.334346.

Zolanvari, S.I., Laefer, D.F. and Natanzi, A.S., 2018. Threedimensional building façade segmentation and opening area detection from point clouds. ISPRS Journal of Photogrammetry and Remote Sensing. 